(C)2009 IEEE. Personal use of this material is permitted. However, permission to reprint/republish this material for advertising or promotional purposes or for creating new collective works for resale or redistribution to servers or lists, or to reuse any copyrighted component of this work in other works must be obtained from the IEEE. 


\title{
Rank Stability Radius for a Matrix with Structured Scalar Perturbations
}

\author{
Wei Xing, Wei-Yong Yan, Wanquan Liu
}

\begin{abstract}
In this paper, the rank stability radius problem is proposed for a real matrix under structured scalar perturbations and some interesting results are achieved based on polynomial analysis. In addition, a computable formula and a two-step procedure are obtained which nicely solves the problem in this simple setup. Finally, these results on rank stability radius are used to estimate the stability robustness of descriptor systems, and for a special class of symmetric descriptor systems, the rank stability radius is proved to be equal to the system stability radius.
\end{abstract}

\section{INTRODUCTION}

$\mathrm{R}$ ANK is one of the most important notions in linear algebra. In several other fields, it is also common to find some important applications of rank technique (e.g., in signal processing see [1], in image processing see [2], etc..) In control systems, for example, many important issues such as controllability and observability [3], input/output decoupling zero [4], impulse-free in descriptor systems [5], reduced-order observer design [6], rank minimization [7], rank constraint [8], etc. can be in part or fully associated with the matrix rank problems. However, it seems that very few results for the problem of rank perturbations have been achieved though perturbations frequently arise in practical systems and the associated problems of stability perturbations have attracted many researchers' attention. This may be partly due to a fact that the problem is hard to deal with or this problem has not brought extensive attention until now. Based on such observations, we will investigate this problem with a simple setup in this paper.

In conventional notion, "stability" implies the ability for an object to maintain its performance under certain extent of perturbations. It is well known that for a continuous linear time-invariant (LTI) system, it is stable if and only if all the eigenvalues of its system matrix have negative real parts. Such matrix is also called Hurwitz matrix. By continuity of eigenvalues to matrix elements, a Hurwitz matrix does have the stability since its entire eigenvalues can still maintain lying in the open left-half complex plane for certain extent of perturbations to its entries.

Manuscript received March 6, 2009

Wei Xing is with the Institute of Systems Science, College of Science, Northeastern University, Shenyang, Liaoning 110004, China (Tel: 8624 83685113; e-mail: awxing@mail.neu.edu.cn).

Wei-Yong Yan is with the Department of Electrical and Computer Engineering, Curtin University of Technology, Perth 6845, Australia (e-mail: w.y.yan@ece.curtin.edu.au)

Wanquan Liu is with the Department of Computing, Curtin University of Technology, Perth 6845, Australia (e-mail: W.Liu@curtin.edu.au).
Similarly for a matrix, if its rank can maintain unchangeable under perturbations, we use the notion of stability and call it to be rank-stable. A matrix may or may not be rank-stable, for example, $\operatorname{diag}(1,1+\delta)$ is rank-stable with respect to perturbation $\delta$ but $\operatorname{diag}(1, \delta)$ is not. Rank stability robustness problem may appear in many systems. For instance, if a Hurwitz matrix has very small rank stability robustness, we can confirm that the corresponding LTI system has very conservative stability robustness. Quantitative measures of rank stability robustness are therefore an important research topic and the rank stability radius is one of such measures. In this paper, we will focus our interests on the rank stability radius under a given structure with scalar perturbations.

Let $A \in \mathbb{R}^{m \times n}$, the rank stability radius of $A$ under a structured matrix $B \in \mathbb{R}^{m \times n}$ with a scalar perturbation $\delta \in \mathbb{R}$ is defined as

$$
\begin{gathered}
\mathfrak{R}(A, B):=\sup \{\gamma \in \mathbb{R}|\forall \delta \in \mathbb{R},| \delta \mid \leq \gamma, \\
\operatorname{rank}(A+\delta B)=\operatorname{rank}(A)\} .
\end{gathered}
$$

An equivalent form of (1) is

$$
\mathfrak{R}(A, B)=\inf \{|\delta| \mid \delta \in \mathbb{R}, \operatorname{rank}(A+\delta B) \neq \operatorname{rank}(A)\} .
$$

This radius measures the largest "possibility" of rank stability robustness under perturbations in (1), it can also be seen as a measure of the smallest "distance", in certain normed linear space, from the point A to a given subset in (2). This radius is not only significant for some robustness problems in system analysis or design, but also has some applications such as in characterization of controllability, observability, etc..

This paper is organized as follows.

In section 2, we will solve the rank stability radius problem. A number of results on characterizations of rank inequalities, properties of the support sets are obtained, and some necessary and/or sufficient conditions for zero radius or infinity radius, and a computable formula for nonzero radius are presented with a two-step procedure.

In section 3, we will use the results obtained in section 2 to estimate the stability robustness for the descriptor systems from a new perspective. The stability radius problem has attracted much attention for years (for normal systems, see [9]-[12]; for descriptor systems, see [13] and [14]), but the known results in descriptor systems are not as explicit as those in normal systems because descriptor system is more complicated and more difficult to deal with due to the impulsive phenomena. In this section, we show that the rank 
stability radius is actually an upper bound of the stability radius for the perturbed descriptor systems, and furthermore in a special type of descriptor systems, the rank stability radius is equal to the stability radius.

In section 4, some numerical examples are given to illustrate the effectiveness of the results in the paper.

\section{MaIn Results}

We first introduce some notations and notions which will be used in this paper.

$$
\Lambda_{m}^{k}:=\left\{\alpha=\left(i_{1}, i_{2}, \cdots, i_{k}\right) \mid 1 \leq i_{1}<i_{2}<\cdots<i_{k} \leq m\right\}
$$

where every $i_{t}, m$ and $k$ are integers with $m \geq k \geq 1$.

Let $A, B \in \mathbb{R}^{m \times n}$, and

$$
\begin{aligned}
& \alpha=\left(i_{1}, i_{2}, \cdots, i_{k}\right) \in \Lambda_{m}^{k} \\
& \beta=\left(j_{1}, j_{2}, \cdots, j_{k}\right) \in \Lambda_{n}^{k}
\end{aligned}
$$

$A(\alpha, \beta)$ denotes the $k \times k$ submatrix of $A$ formed by rows $i_{1}, i_{2}, \cdots, i_{k}$ of $A$ and columns $j_{1}, j_{2}, \cdots, j_{k}$ of $A$; and $A(\alpha, \beta)^{c}$ denotes the complementary submatrix of $A(\alpha, \beta)$ in $A$.

Let

$$
\begin{gathered}
A[k]:=\sum_{\alpha \in \Lambda_{m}^{k}, \beta \in \Lambda_{n}^{k}}(\operatorname{det} A(\alpha, \beta))^{2} \\
\varphi_{-}(x):=(A+x B)[\operatorname{rank}(A)] \\
\varphi_{+}(x):=(A+x B)[\operatorname{rank}(A)+1]
\end{gathered}
$$

where $x$ is a scalar. One can see easily that only when $\operatorname{rank}(A)<\min \{m, n\}, \varphi_{+}(x)$ exists. Further define

$$
\begin{aligned}
& \Omega^{-}:=\{\delta \in \mathbb{R} \mid \operatorname{rank}(A+\delta B)<\operatorname{rank}(A)\}, \\
& \Omega^{+}:=\{\delta \in \mathbb{R} \mid \operatorname{rank}(A+\delta B)>\operatorname{rank}(A)\} .
\end{aligned}
$$

For $A, B \in \mathbb{R}^{n \times n}$, the matrix pair $(A, B)$ is said to be regular if $\operatorname{det}(x A-B)$, as a polynomial with respect to $x$, is nonzero. and

$$
\sigma(A, B):=\{s \in \mathbb{C} \mid \operatorname{det}(s A-B)=0\}
$$

is the spectrum of pair $(A, B)$. Specially, $\sigma(B):=\sigma(I, B)$ is the spectrum of $B$.

Obviously, those defined by (3)-(7) have the following basic properties:

1) $A[1]^{1 / 2}$ is precisely Frobenius norm of $A$;

2) $\varphi_{-}(x)$ and $\varphi_{+}(x)$ are polynomials with respect to $x$ and $\varphi_{-}(x) \neq 0$;

3) $\Omega^{-}$and $\Omega^{+}$are support sets for rank changes, and $\Omega^{-} \cap \Omega^{+}=\varnothing, 0 \notin \Omega^{-} \cup \Omega^{+}$.

With (3), one can prove the following characterizations of some rank inequalities.

Lemma 1 Let $A \in \mathbb{R}^{m \times n}, r$ be positive integer. Then

1) $\operatorname{rank}(A)<r \Leftrightarrow A[r]=0$;

2) $\operatorname{rank}(A)>r \Leftrightarrow A[r+1]>0$.

Theorem 1 Let $A, B \in \mathbb{R}^{m \times n}$. Then

$$
\text { 1) } \Omega^{-}=\left\{\delta \in \mathbb{R} \mid \varphi_{-}(\delta)=0\right\} \text {; }
$$

2) $\Omega^{+}=\left\{\delta \in \mathbb{R} \mid \varphi_{+}(\delta)>0\right\}$.

Corollary 1 Let $A, B \in \mathbb{R}^{m \times n}$. Then

1) $\Omega^{-}=\varnothing$ or $\Omega^{-}$is a finite set;

2) $\Omega^{+}=\varnothing$ or $\Omega^{+}$is an open set.

These results are based on the following facts:

1) $\Omega^{-}$consists of all real roots of $\varphi_{-}(x)$;

2) If $\varphi_{+}(x)$ exists and $\varphi_{+}(x) \neq 0, \Omega^{+}$is an open set by continuity.

With the above description of support sets, we can characterize the rank stability radius now.

Theorem 2 Let $A, B \in \mathbb{R}^{m \times n}$. Then

1) $\mathfrak{R}(A, B)=0 \Leftrightarrow \Omega^{+} \neq \varnothing$;

2) $\mathfrak{R}(A, B)=+\infty \Leftrightarrow \Omega^{+}=\varnothing$ and $\Omega^{-}=\varnothing$;

3) $0<\Re(A, B)<+\infty \Leftrightarrow \Omega^{+}=\varnothing$ and $\Omega^{-} \neq \varnothing$.

Proof. 1) By (2), if $\mathfrak{R}(A, B)=0$, there exists a sequence of real numbers $\left\{\delta_{i}\right\} \subset\left(\Omega^{-} \cup \Omega^{+}\right)$such that

$$
\delta_{i} \rightarrow 0 \text { as } i \rightarrow+\infty \text {. }
$$

Since $\Omega^{-}$is finite, for some sufficient larger $i, \delta_{i} \notin \Omega^{-}$, hence $\delta_{i} \in \Omega^{+}$.

Conversely, if $\Omega^{+} \neq \varnothing$, there is $\delta \in \mathbb{R}$ such that $\varphi_{+}(\delta)>0$, which implies that the polynomial $\varphi_{+}(x) \neq 0$, thus $\varphi_{+}(x)$ has only a finite number of roots. Let us denote the nonzero real root nearest to 0 by $\delta_{0}$, then for any $\delta \in\left(0,\left|\delta_{0}\right|\right), \varphi_{+}(\delta)>0$, hence $\mathfrak{R}(A, B)=0$.

2) By (1),

$$
\begin{aligned}
& \Re(A, B)=+\infty \\
& \Leftrightarrow \forall \delta \in \mathbb{R}, \operatorname{rank}(A+\delta B)=\operatorname{rank}(A) \\
& \Leftrightarrow \forall \delta \in \mathbb{R}, \delta \notin \Omega^{-} \text {and } \delta \notin \Omega^{+} \\
& \Leftrightarrow \Omega^{-}=\varnothing \text { and } \Leftrightarrow \Omega^{+}=\varnothing .
\end{aligned}
$$

3 ) It can be directly derived from 1) and 2).

Remark 1 By theorem 2, the rank stability radius depends on the different cases of the support sets. Among these cases, whether $\Omega^{+} \neq \varnothing$ is an important condition. If such case occurs, $\mathfrak{R}(A, B)=0$, which indicates that there is no robustness for rank perturbations.

Next we characterize the condition $\Omega^{+} \neq \varnothing$.

Theorem 3 Let $A, B \in \mathbb{R}^{m \times n}$. Then $\Omega^{+} \neq \varnothing \Leftrightarrow \operatorname{rank}(A)<$ $\min \{m, n\}$ and there exist $\alpha \in \Lambda_{m}^{\operatorname{rank}(A)+1}$, and $\beta \in \Lambda_{n}^{\operatorname{rank}(A)+1}$, such that the matrix pair $(B(\alpha, \beta), A(\alpha, \beta))$ is regular.

Proof. By theorem 1, $\Omega^{+} \neq \varnothing \Leftrightarrow \varphi_{+}(x)$ is nonzero $\Leftrightarrow \operatorname{rank}(A)<\min \{m, n\}$ and there exist $\alpha \in \Lambda_{m}^{\operatorname{rank}(A)+1}$ and $\beta \in \Lambda_{n}^{\operatorname{rank}(A)+1}$ such that

$$
\operatorname{det}(A(\alpha, \beta)+x B(\alpha, \beta))=\operatorname{det}(A+x B)(\alpha, \beta) \neq 0
$$

which is equivalent to that $(B(\alpha, \beta), A(\alpha, \beta))$ is regular.

To give another characterization of $\Omega^{+} \neq \varnothing$, we introduce 
the following lemma.

Lemma 2 Let $A, B \in \mathbb{R}^{n \times n}$. Then

$$
\begin{gathered}
\operatorname{det}(A+x B)=x^{n} \operatorname{det} B+\operatorname{det} A \\
+\sum_{k=1}^{n-1} x^{k}\left(\sum_{\alpha, \beta \in \Lambda_{n}^{k}}(-1)^{(\alpha+\beta) u} \operatorname{det} B(\alpha, \beta) \operatorname{det} A(\alpha, \beta)^{c}\right)
\end{gathered}
$$

where $u:=(1,1, \cdots, 1)^{T} \in \mathbb{R}^{k}, T$ represents transpose.

Theorem 4 Let $A, B \in \mathbb{R}^{m \times n}$. Then $\Omega^{+} \neq \varnothing \Leftrightarrow \operatorname{rank}(A)<$ $\min \{m, n\}$ and there exist $\alpha \in \Lambda_{m}^{\operatorname{rank}(A)+1}, \beta \in \Lambda_{n}^{\operatorname{rank}(A)+1}$, and $1 \leq k \leq \operatorname{rank}(A)+1$ such that

$$
\begin{gathered}
\sum_{\eta, \xi \in \Lambda_{\text {rank }(A)+1}^{k}}(-1)^{(\eta+\xi) u} \operatorname{det}(B(\alpha, \beta))(\eta, \xi) \\
\cdot \operatorname{det}(A(\alpha, \beta))(\eta, \xi)^{c} \neq 0
\end{gathered}
$$

where $\quad u=(1,1, \cdots, 1)^{T} \in \mathbb{R}^{k} \quad$ and $\quad \operatorname{det}(A(\alpha, \beta))(\eta, \xi)^{c}=1$ whenever $k=\operatorname{rank}(A)+1$.

Proof. Since $\varphi_{+}(x)$ exists $\Leftrightarrow \operatorname{rank}(A)<\min \{m, n\}$, and $\varphi_{+}(x) \neq 0 \Leftrightarrow$ there exist $\alpha \in \Lambda_{m}^{\operatorname{rank}(A)+1}$ and $\beta \in \Lambda_{n}^{\operatorname{rank}(A)+1}$, such that

$$
\operatorname{det}(A+x B)(\alpha, \beta) \neq 0 .
$$

But by lemma 2,

$$
\begin{gathered}
\operatorname{det}(A+x B)(\alpha, \beta)=\operatorname{det}(A(\alpha, \beta)+x B(\alpha, \beta)) \\
=x^{\operatorname{rank}(A)+1} \operatorname{det} B(\alpha, \beta)+\sum_{k=1}^{\operatorname{rank}(A)} x^{k} \\
\left.\cdot\left(\sum_{\eta, \xi \in \Lambda_{\text {rank }(A)+1}^{k}}(-1)^{(\eta+\xi) u} \operatorname{det}(B(\alpha, \beta))(\eta, \xi)\right) \operatorname{det}(A(\alpha, \beta))(\eta, \xi)^{c}\right)
\end{gathered}
$$

Therefore, (9) holds $\Leftrightarrow$ the polynomial (10) has at least one nonzero coefficient. Thus, the proof is completed.

Note that, if $\operatorname{rank}(B)>\operatorname{rank}(A)$, there must exist a nonzero minor of order $\operatorname{rank}(A)+1$ of $B$, namely $\operatorname{det} B(\alpha, \beta) \neq 0$, by theorem $4, \Omega^{+} \neq \varnothing$. Thus, a sufficient condition for $\mathfrak{R}(A, B)=0$ is obtained as follows.

Corollary 2 Let $A, B \in \mathbb{R}^{m \times n}$. If $\operatorname{rank}(B)>\operatorname{rank}(A)$, then $\mathfrak{R}(A, B)=0$.

Next we intend to provide a necessary condition for $\mathfrak{R}(A, B)=0$.

Proposition 1 Let $A, B \in \mathbb{R}^{m \times n}$. If $\mathfrak{R}(A, B)=0$, then

$$
\operatorname{rank}([A, B])>\operatorname{rank}(A) \text {. }
$$

Proof. By theorem 2, since $\Omega^{+} \neq \varnothing$, there exists $\delta \in \mathbb{R}$ such that $\operatorname{rank}(A+\delta B)>\operatorname{rank}(A)$. However,

$$
A+\delta B=[A, B]\left[\begin{array}{c}
I \\
\delta I
\end{array}\right]
$$

and

$$
\operatorname{rank}([A, B]) \geq \operatorname{rank}(A+\delta B)
$$

Hence

$$
\operatorname{rank}([A, B])>\operatorname{rank}(A)
$$

Remark 2 In proposition $1, \operatorname{rank}([A, B])>\operatorname{rank}(A)$ can not serve as a sufficient condition for $\mathfrak{R}(A, B)=0$. A counter example is constructed below.

Let

$$
A=\left[\begin{array}{ll}
1 & 1 \\
0 & 0
\end{array}\right], B=\left[\begin{array}{ll}
1 & 1 \\
1 & 1
\end{array}\right]
$$

Then

$$
\operatorname{rank}([A, B])>\operatorname{rank}(A)
$$

but $\Omega^{+}=\varnothing$, i.e., $\mathfrak{R}(A, B)>0$. Similarly, one can easily verify that the inverse statement of corollary 2 is not true.

We now turn our attention to characterizing set $\Omega^{-}$. From theorem 1, one can easily derive the following.

Theorem 5 Let $A, B \in \mathbb{R}^{m \times n}$. Then

$$
\Omega^{-}=\bigcap_{\alpha \in \Lambda_{m}^{\operatorname{rank}(A)}, \beta \in \Lambda_{n}^{\operatorname{rank}(A)}} \sigma(B(\alpha, \beta), A(\alpha, \beta)) \cap \mathbb{R} .
$$

Corollary 3 Let $A, B \in \mathbb{R}^{m \times n}$. Then $\Omega^{-}=\varnothing \Leftrightarrow$

$$
\bigcap_{\alpha \in \Lambda_{m}^{\operatorname{rank}(A)}, \beta \in \Lambda_{n}^{\operatorname{rank}(A)}} \sigma(B(\alpha, \beta), A(\alpha, \beta)) \cap \mathbb{R}=\varnothing .
$$

This corollary can be sometimes very effective in practice. For example, if we observe $B(\alpha, \beta)=0$ but $\operatorname{det} A(\alpha, \beta) \neq 0$ for some $\alpha \in \Lambda_{m}^{\operatorname{rank}(A)}$ and $\beta \in \Lambda_{n}^{\operatorname{rank}(A)}$, then we can conclude at once that $\Omega^{-}=\varnothing$ because $\sigma(B(\alpha, \beta), A(\alpha, \beta))=\varnothing$. In the following example with

$$
\begin{aligned}
A & =\left[\begin{array}{cccc}
10 & 2 & 4 & 5 \\
-1 & 2 & 1 & 2 \\
9 & 4 & 5 & 7
\end{array}\right] \\
B & =\left[\begin{array}{cccc}
25 & 0 & 1 & 0 \\
-1 & 1 & 75 & 1 \\
27 & 0 & -1 & 0
\end{array}\right]
\end{aligned}
$$

$\operatorname{rank}(A)=2, B((1,3),(2,4))=0$, but $\operatorname{det} A((1,3),(2,4)) \neq 0$, thus

$$
\sigma(B((1,3),(2,4)), A((1,3),(2,4)))=\varnothing
$$

and (12) holds, hence $\Omega^{-}=\varnothing$.

Now we give a general characterization for the nonzero and finite rank stability radius.

Theorem 6 Let $A, B \in \mathbb{R}^{m \times n}, \Omega^{+}=\varnothing$ and $\Omega^{-} \neq \varnothing$. Then

$$
\begin{aligned}
\mathfrak{R}(A, B) & =\min \left\{|\delta| \mid \delta \in \Omega^{-}\right\} \\
& =\min \left\{|\delta| \mid \delta \in \mathbb{R}, \varphi_{-}(\delta)=0\right\} .
\end{aligned}
$$

Proof. There exists $\delta_{0} \in \Omega^{-}$such that

$$
\left|\delta_{0}\right|=\min \left\{|\delta| \mid \delta \in \Omega^{-}\right\}>0 .
$$

Since $\Omega^{+}=\varnothing, \forall \delta \in \mathbb{R}$ with $0 \leq|\delta|<\left|\delta_{0}\right|$,

$$
\operatorname{rank}(A+\delta B)=\operatorname{rank}(A)
$$

therefore, $\mathfrak{R}(A, B)=\left|\delta_{0}\right|$, (13) holds and (14) also holds by 
theorem 1 .

If $A, B \in \mathbb{R}^{m \times n}$ with $A$ full rank, i.e., $\operatorname{rank}(A)=\min \{m, n\}$, then $\varphi_{+}(x)$ does not exist, $\Omega^{+}=\varnothing, \mathfrak{R}(A, B)>0$. If the matrices are further square, then $\varphi_{-}(x)=(\operatorname{det}(A+x B))^{2}$, $\varphi_{-}(\delta)=0 \Leftrightarrow \delta^{-1} \in \sigma\left(-A^{-1} B\right) \backslash\{0\}$. This derivation gives the following theorem.

Theorem 7 Let $A, B \in \mathbb{R}^{n \times n}$ with $A$ nonsingular. Then

$$
\mathfrak{R}(A, B)=\frac{1}{\max \left\{|\delta| \mid \delta \in \sigma\left(A^{-1} B\right) \cap \mathbb{R}\right\}}
$$

where $\mathfrak{R}(A, B)=+\infty$ whenever $\sigma\left(A^{-1} B\right) \cap \mathbb{R}=\{0\}$ or $\varnothing$.

Based on previous investigations, we can now present the following two-step procedure for computing the rank stability radius:

Step 1. Compute and test if $\Omega^{+} \neq \varnothing$. If so, $\mathfrak{R}(A, B)=0$; otherwise, go to step 2.

Step 2. Compute and test if $\Omega^{-}=\varnothing$. If so, $\mathfrak{R}(A, B)=+\infty$; otherwise, $\mathfrak{R}(A, B)=\min \left\{|\delta| \mid \delta \in \mathbb{R}, \varphi_{-}(\delta)=0\right\} \quad$ (or using (15), or (13) with (11)).

\section{APPLICATION IN DESCRIPTOR SYSTEMS}

A typical homogeneous LTI descriptor system is modeled as

$$
E \dot{x}(t)=A x(t)
$$

where $E, A \in \mathbb{R}^{n \times n}, x(t) \in \mathbb{R}^{n}$ is state. If $\operatorname{rank}(E)=n$, the system becomes a normal LTI system. A descriptor system is, in general, required to be regular, i.e., $(E, A)$ is regular. The system (16) is said to be stable if $\sigma(E, A) \subset \mathbb{C}^{-}$, where $\mathbb{C}^{-}:=\{s \in \mathbb{C} \mid \operatorname{Re}(s)<0\}$.

Consider the following perturbed system with scalar perturbation occurred within the system

$$
E \dot{x}(t)=(A+\delta B) x(t)
$$

where $\delta \in \mathbb{R}$ is an unknown perturbation, $B \in \mathbb{R}^{n \times n}$ is a known matrix representing the perturbation structure. Assume the nominal system (16) is stable, then the stability radius of the perturbed system (17) with respect to perturbation $\delta$ is defined as

$$
\Im(E, A, B):=\inf \left\{|\delta| \mid \delta \in \mathbb{R}, \sigma(E, A+\delta B) \not \subset \mathbb{C}^{-}\right\} .
$$

For normal systems, the system stability radius problem has been studied for over two decades, the formulas for computing radii have been achieved ([9]-[11]). However, in descriptor systems, such problems are far from being resolved ([13], [14]) because the descriptor systems are more complicated.

With the method of rank stability radius, we can give an upper bound on the stability radius.

Theorem 8 Let system (16) be stable, $\mathfrak{I}(E, A, B)$ be the stability radius of the system (17). Then

$$
\Im(E, A, B) \leq \mathfrak{R}(A, B) .
$$

Proof. Since system (16) is stable, $A$ is nonsingular, from theorem $3, \Omega^{+}=\varnothing$, i.e., $\mathfrak{R}(A, B)>0$.

If $\mathfrak{R}(A, B)=+\infty$, the inequality (19) holds naturally; otherwise, $\Omega^{-} \neq \varnothing$, by theorem 6 , there exists $\delta_{0} \in \Omega^{-}$such that $\left|\delta_{0}\right|=\mathfrak{R}(A, B)$, and

$$
\operatorname{rank}\left(A+\delta_{0} B\right)<\operatorname{rank}(A)
$$

thus $\operatorname{det}\left(A+\delta_{0} B\right)=0$, therefore, $0 \in \sigma\left(E, A+\delta_{0} B\right)$ and $\sigma\left(E, A+\delta_{0} B\right) \not \subset \mathbb{C}^{-}$, hence,

$$
\mathfrak{J}(E, A, B) \leq\left|\delta_{0}\right|=\mathfrak{R}(A, B) .
$$

The rank stability radius (1) is an upper bound of the stability radius (18) (this upper bound is also that of the stability radius for a normal system, $E=I$ in (17). The inequality (19) can be used to estimate the stability robustness of a system. Moreover, for a special class of descriptor systems, the rank stability radius can be equal to the stability radius.

The system (16) is said to be symmetric if $E=E^{T}$ and $A=A^{T}$. Symmetric descriptor systems have many practical applications (e.g., see [15]).

Theorem 9 Let system (16) be symmetric.

1) If $A>0$ then, the system is stable $\Leftrightarrow E \leq 0$;

2) If $A<0$ then, the system is stable $\Leftrightarrow E \geq 0$.

where $A>0(\geq 0,<0, \leq 0)$ implies that $A$ is positive definite (positive semi-definite, negative, negative semi-definite), respectively.

Proof. The proof is given only for the case of $A>0$. There exists a nonsingular matrix $P$ such that $P^{T} A P=I$, then

$$
s \in \sigma(E, A) \Leftrightarrow s^{-1} \in \sigma\left(P^{T} E P\right) \backslash\{0\}
$$

Hence

$$
\begin{aligned}
\sigma(E, A) \subset \mathbb{C}^{-} & \Leftrightarrow \sigma\left(P^{T} E P\right) \backslash\{0\} \subset \mathbb{C}^{-} \\
& \Leftrightarrow E \leq 0 .
\end{aligned}
$$

A symmetric system (16) with $A>0$ or $A<0$ is said to be definite. A definite system has good nature, e.g., there is no impulsive behaviour for such system, and further $\sigma(E, A) \subset \mathbb{R}$ if system (16) is definite.

Lemma 3 Let $A, B \in \mathbb{R}^{n \times n}$ with $A>0(A<0)$ and $B=B^{T} \neq 0$. Then there exists $\delta_{0} \in \mathbb{R}$ such that $A+\delta_{o} B$ is not positive definite (negative definite).

Theorem 10 Let system (16) be definite and stable. Then for any $B \in \mathbb{R}^{n \times n}$ with $B=B^{T}$,

$$
\mathfrak{I}(E, A, B)=\mathfrak{R}(A, B)<+\infty .
$$

Proof. The proof is given only for the case of $A>0$.

From stability of system (16) and theorem $9, E \leq 0$. If $B=0$, it is trivial. If $B \neq 0$, let

$$
M(\delta):=A+\delta B \text {. }
$$

Since $M(0)>0$, by the continuity of eigenvalues and 
lemma 3, there exists a real finite number $\gamma>0$ such that as long as $|\delta|<\gamma, M(\delta)>0$ holds, but for some $\delta_{0} \in \mathbb{R}$ with $\left|\delta_{0}\right|=\gamma, M\left(\delta_{0}\right)$ has at least one zero eigenvalue. Hence, for $|\delta|<\gamma$, system (17) is stable by theorem 9, for $\delta=\delta_{0}$, if (17) is regular, it must be unstable, so $\mathfrak{I}(E, A, B)=\gamma$.

Since $|\delta|<\gamma, M(\delta)>0$, i.e., $\operatorname{rank}(A+\delta B)=\operatorname{rank}(A)$; $\delta=\delta_{0}, \operatorname{det} M\left(\delta_{0}\right)=0$ implies $\operatorname{rank}\left(A+\delta_{0} B\right)<\operatorname{rank}(A)$, therefore, $\mathfrak{R}(A, B)=\gamma$ by (2).

Remark 3 Theorem 10 will fail if the system loses the definiteness. A counterexample is given as follows. Let

$$
E=\operatorname{diag}(1,-1,0), A=\left[\begin{array}{ccc}
-1 & -1 & 1 \\
-1 & 1 & -1 \\
1 & -1 & -1
\end{array}\right]
$$

then the system (16) is symmetric. Since

$$
\sigma(E, A)=\{-1-\sqrt{3} i,-1+\sqrt{3} i\}
$$

and

$$
\sigma(A)=\{-2,-1,2\}
$$

the system (16) is stable but not definite. Consider the corresponding perturbed system (17) with $B=\operatorname{diag}(0,1,-1)$. One can derive the following easily

$$
\begin{aligned}
f(\delta, \lambda) & :=\operatorname{det}(\lambda E-(A+\delta B)) \\
& =-(\delta+1) \lambda^{2}-(\delta+1)(\delta+2) \lambda-\left(\delta^{2}+2 \delta+4\right)
\end{aligned}
$$

where $\delta \in \mathbb{R}$. The two roots of $f(\delta, \lambda)$ with respect to $\lambda$ are

$$
\lambda_{1,2}=\frac{-1}{2(\delta+1)}\left((\delta+1)(\delta+2) \pm \sqrt{(\delta+1)\left(\delta^{3}+\delta^{2}-12\right)}\right) .
$$

With $\delta=-1, f(-1, \lambda)=-3, \sigma(E, A-B)=\varnothing$, the corresponding system $(17)$ is regular but unstable.

With $|\delta|<1, \operatorname{Re}\left(\lambda_{1,2}\right)=-(\delta+2) / 2<0$, the system is stable.

Thus, $\mathfrak{I}(E, A, B)=1$ by (18). However, since $A$ is nonsingular and

$$
\sigma\left(A^{-1} B\right)=\{0,0.2500-0.4330 i, 0.2500+0.4330 i\}
$$

by theorem $7, \mathfrak{R}(A, B)=+\infty$.

\section{ILLUSTRATIVE NUMERICAL EXAMPLES}

In this section, we provide several examples to illustrate some main results.

Example 1 1) Let

$$
A=\operatorname{diag}(1,0), B=I_{2}
$$

then $A+\delta B=\operatorname{diag}(1+\delta, \delta), \Omega^{+} \neq \varnothing, \mathfrak{R}(A, B)=0$ by theorem 2 . Using corollary 2 , one can directly lead to $\mathfrak{R}(A, B)=0$.

2) Let

$$
A=\operatorname{diag}(1,0), B=\left[\begin{array}{ll}
0 & 1 \\
0 & 0
\end{array}\right]
$$

then

$$
A+\delta B=\left[\begin{array}{ll}
1 & \delta \\
0 & 0
\end{array}\right]
$$

and $\Omega^{+}=\Omega^{-}=\varnothing, \mathfrak{R}(A, B)=+\infty$ by theorem 2 . Also, here $\Omega^{+}=\varnothing$ can be derived from $\operatorname{rank}([A, B]) \leq \operatorname{rank}(A)$ by proposition 1 , and $\varphi_{-}(x)=x^{2}+1$ implies that $\Omega^{-}=\varnothing$ by theorem 1.

3) Consider

$$
A=I_{2}, B=\operatorname{diag}(1,2)
$$

Then

$$
A+\delta B=\operatorname{diag}(1+\delta, 1+2 \delta)
$$

$\Omega^{+}=\varnothing, \Omega^{-}=\{-1,-1 / 2\}$, so $\mathfrak{R}(A, B)=1 / 2$. Alternatively, since $A$ is nonsingular, $\sigma\left(A^{-1} B\right)=\sigma(B)=\{1,2\}$, by $(15)$, $\mathfrak{R}(A, B)=1 / 2$.

Example 2 1) Let

$$
\begin{gathered}
A=\left[\begin{array}{ccccc}
13 & -7 & -15 & 2 & 5 \\
9 & 11 & 3 & -4 & 0 \\
-5 & -29 & -21 & 10 & 5
\end{array}\right], \\
B=\left[\begin{array}{ccccc}
0 & 1 & -1 & 0 & 2 \\
3 & 1 & 0 & 1 & -2 \\
3 & -1 & 2 & 1 & -6
\end{array}\right] .
\end{gathered}
$$

Then $\operatorname{rank}(A)=2$, after taking $\alpha=(1,2,3), \beta=(2,4,5)$, $k=2$, the left side of (8) is equal to 195 , by theorem 4 , $\mathfrak{R}(A, B)=0$.

2) Let

$$
\begin{aligned}
A & =\left[\begin{array}{cccc}
9 & 1 & 32 & 1 \\
4 & 0 & -2 & 1 \\
13 & 1 & 30 & 2
\end{array}\right], \\
B & =\left[\begin{array}{cccc}
-2 & 1 & -3 & 0 \\
11 & 2 & 15 & 1 \\
9 & 3 & 12 & 1
\end{array}\right] .
\end{aligned}
$$

Then $\operatorname{rank}([A, B])=\operatorname{rank}(A)=2$, by proposition 1 , one has $\Omega^{+}=\varnothing$. Also $\sigma(B((1,2),(2,4)), A((1,2),(2,4)))=\{-i, i\}$, therefore (12) holds, i.e., $\Omega^{-}=\varnothing$. Hence $\mathfrak{R}(A, B)=+\infty$ by theorem 2.

3) Consider

$$
\begin{aligned}
& A=\left[\begin{array}{ccc}
0.0153 & -0.9318 & -0.8462 \\
-0.7468 & -0.4660 & 0.5252 \\
0.4451 & -0.4186 & 0.2026
\end{array}\right], \\
& B=\left[\begin{array}{ccc}
-0.6721 & 0.6813 & -0.5028 \\
0.8381 & -0.3795 & 0.7095 \\
0.0196 & -0.8318 & -0.4289
\end{array}\right] .
\end{aligned}
$$

Then $\operatorname{rank}(A)=3$ and

$$
\sigma\left(A^{-1} B\right)=\{-0.1323,0.2524+0.9157 i, 0.2524-0.9157 i\} \text {. }
$$


By theorem 7, $\Re(A, B)=1 / 0.1323=7.5586$.

Example 3 1) Let

$$
\begin{aligned}
E & =\left[\begin{array}{ccc}
16 & 11 & 31 \\
-2 & 5 & -6 \\
6 & 6 & 11
\end{array}\right], \\
A & =\left[\begin{array}{ccc}
18 & 47 & 134 \\
28 & 13 & 84 \\
25 & 45 & 136
\end{array}\right], \\
B & =\left[\begin{array}{ccc}
21 & 2 & 31 \\
8 & -13 & -6 \\
11 & -3 & 11
\end{array}\right] .
\end{aligned}
$$

Since $\sigma(E, A)=\{-1,-2\} \subset \mathbb{C}^{-}$, the system $(16)$ is stable. As a polynomial with respect to $\lambda, \operatorname{det}(\lambda E-(A+\delta B))$ has two roots: $\lambda_{1}=\delta-1, \lambda_{2}=-\delta-2$, so the perturbed system (17) is stable if and only if $-2<\delta<1$. When $\delta=1$, the system (17) is regular but not stable, hence $\mathfrak{I}(E, A, B)=1$ by (18).

Since $A$ is nonsingular and

$$
\sigma\left(A^{-1} B\right)=\{-1,0,1 / 2\}
$$

from the formula (15) we have $\mathfrak{R}(A, B)=1$, thus, $\mathfrak{I}(E, A, B)=\mathfrak{R}(A, B)$, the estimation of stability radius of the system (17) in theorem 8 is exact.

2) Let

$$
\begin{aligned}
E & =\left[\begin{array}{ccc}
1 & -3 & 2 \\
-3 & 9 & -6 \\
2 & -6 & 4
\end{array}\right], \\
A & =\left[\begin{array}{ccc}
-5 & 1 & -2 \\
1 & -2 & 1 \\
-2 & 1 & -3
\end{array}\right], \\
B & =\left[\begin{array}{ccc}
1 & 2 & -3 \\
2 & -2 & 0 \\
-3 & 0 & 3
\end{array}\right] .
\end{aligned}
$$

Since $E \geq 0$ (with $\operatorname{rank}(E)=1$ ) and $A<0$, from theorem 9, the system (16) is stable. $B$ is indefinite and singular, by theorem 10 and (15), the stability radius of perturbed system (17) is

$$
\begin{aligned}
\Im(E, A, B) & =\mathfrak{R}(A, B) \\
& =0.3425 .
\end{aligned}
$$

\section{CONCLUSIONS}

Firstly, we have discussed the rank stability radius problem and obtained a number of results which include:

1) the rank inequality characterizations;

2) properties of support sets;

3) some necessary and/or sufficient conditions for zero and infinity radius, respectively;

4) a formula for nonzero radius;
5) a two-step procedure for computing the rank stability radius.

Secondly, we have used the rank stability radius to estimate the stability robustness of the descriptor systems, and showed that the rank stability radius is an upper bound of the stability radius for descriptor systems. In some class of symmetric descriptor systems, we have showed that the rank stability radius is exactly the stability radius.

\section{REFERENCES}

[1] J. H. Manton, R. Mahony, and Y. Hua, "The geometry of weighted low-rank approximations," IEEE Transactions on Signal Processing, vol. 51, pp. 500-514, 2003.

[2] P. Sussner and G. X. Ritter, "Rank-based decompositions of morphological templates," IEEE Transactions on Image Processing, vol. 9, pp. 1420-1430, 2000.

[3] K. Zhou with J. C. Doyle, Essential of Robust Control, Prentice Hall, Upper Saddle River, 1998.

[4] H. H. Rosenbrock, State-Space and Multivariable Theory, Wiley-Interscience, New York, 1970.

[5] L. Dai, Singular Control Systems, Lecture notes in control and information sciences, Springer, Berlin, 1989.

[6] M. Darouach, M. Zasadzinski and M. Hayar, "Reduced-order observer design for descriptor systems with unknown inputs," IEEE Transactions on Automatic Control, vol. 41, pp. 1068-1072, 1996.

[7] M. Mesbahi, "On the rank minimization problem and its control applications," Systems and Control Letters, 33, pp. 31-36, 1998.

[8] D. Henrion, S. Tarbourech, and M. Sebek, "Rank-one LMI approach to simultaneous stabilization of linear systems," Systems and Control Letters, 38, pp. 79-89, 1999.

[9] D. Hinrichsen and A. J. Pritchard, "Stability radii of linear systems," Systems \& Control Letters, 7, pp. 71-10, 1986.

[10] D. Hinrichsen and A. J. Pritchard, "Stability radius for structured perturbations and the algebraic Riccati equation, ”Systems \& Control Letters, 8, pp. 105-113, 1986.

[11] L. Qiu, B. Bernhardsson, A. Rantzer, E. J. Davison, P. M. Young, and J. C. Doyle, "A formula for computation of the real stability radius," Automatica, 31, pp. 879-890, 1995.

[12] W. Y. Yan and J. Lam, "On computation of the stability radius for nonlinearly structured perturbations," Systems \& Control Letters, 34, pp. 273-280, 1998.

[13] R. Byers and N. K. Nichols, "On the stability radius of generalized state-space systems," Linear Algebra and Its Applications, 188/189, pp. 113-134.

[14] Y. Z. Hu and E. J. Davison, "A study of the stability radius for descriptor systems," Proceedings of the 35th Conference on Decision and Control, Kobe, December 1996.

[15] D. Liu and P. J. Antsaklis (Eds.), Stability and Control of Dynamical Systems with Applications, Birkhauser, Boston, 2003. 